\title{
EVALUATION OF THE PROTECTIVE EFFECT OF NIGELLA SATIVA (BLACK CUMIN) OIL AGAINST VANCOMYCIN-INDUCED NEPHROTOXICITY IN RATS
}

\author{
SARAH T OMRAN ${ }^{1 *}$, JAWAD H AHMED \\ ${ }^{1}$ Department of Pharmacology and Toxicology, College of Pharmacy, University of Basrah, Basra, Iraq, ${ }^{2}$ Department of Pharmacology, \\ College of Medicine, University of Basrah, Iraq. Email: saraaltemimi@gmail.com.
}

Received: 24 November 2018, Revised and Accepted: 24 January 2019

\begin{abstract}
Objective: The study was designed to investigate a possible protective effect of Nigella sativa (NS) against vancomycin (VAN)-induced nephrotoxicity in rats.

Methods: Twenty-eight adult male Albino rats were randomly divided into four groups; seven rats in each. Group I (control): The animals were treated with normal saline ( $2 \mathrm{ml} / \mathrm{kg} /$ day) given orally and intraperitoneally (IP); Group II: VAN was given at a dose of $400 \mathrm{mg} / \mathrm{kg} / \mathrm{day}$ for 7 days IP and normal saline orally; Group III: NS oil was given at a dose of $2 \mathrm{ml} / \mathrm{kg} /$ day for seven days orally and normal saline IP; and Group IV: VAN $400 \mathrm{mg} / \mathrm{kg} / \mathrm{day}$ IP in combination with NS oil $2 \mathrm{ml} / \mathrm{kg} /$ day orally for 7 days. Twenty-four hours after the last dose, the animals were sacrificed, and serum was collected to estimate urea and creatinine. Then, both kidneys were excised, one for homogenate preparation to estimate renal tissue malondialdehyde (MDA), glutathione (GSH) and neutrophil gelatinase-associated lipocalin (NGAL) and the other for histopathological examination.
\end{abstract}

Results: NS significantly decreased serum urea and creatinine compared to VAN treated group, p $<0.001$. NS significantly increased renal tissue GSH compared to VAN treated group $\mathrm{p}<0.001$. NS lowered MDA and NGAL levels in the homogenate of renal tissues compared to their elevated levels in rats treated with VAN, but this did not achieve statistical significance. NS also ameliorated renal histopathological changes induced by VAN.

Conclusion: NS has a protective effect against VAN-induced nephrotoxicity.

Keywords: Nigella sativa, Vancomycin, Nephrotoxicity, Oxidative stress.

(C) 2019 The Authors. Published by Innovare Academic Sciences Pvt Ltd. This is an open access article under the CC BY license (http://creativecommons. org/licenses/by/4. 0/) DOI: http://dx.doi.org/10.22159/ajpcr.2019.v12i3.30599

\section{INTRODUCTION}

Vancomycin (VAN) is a glycopeptide antibiotic. It is an exceptionally important antibiotic which should be deserved for the treatment of severe infections such as methicillin-resistant Staphylococcus aureus (MRSA) [1]. VAN, in that context, is effective, but there are numerous side effects encountered with its use. Nephrotoxicity is among these side effects; it is dose and duration of use related and is practically unavoidable [2]. The incidence of VAN-induced nephrotoxicity was reported as (5-35\%) according to different nephrotoxicity definitions [3]. The exact mechanism of VAN-induced nephrotoxicity is not fully known, but previous experimental studies revealed pro-inflammatory oxidation, mitochondrial dysfunction, and cellular apoptosis as the main mechanisms of renal injury [4].

VAN may increase oxidative phosphorylation in renal tubular cells, leading to the formation of superoxide, especially in the mitochondria. Furthermore, activation of caspase 6 in the presence of superoxide inside the mitochondria may lead to peroxidation of mitochondrial membrane phospholipids and specially cardiolipin leading to changes in mitochondrial membrane potential (depolarization) and an increase in the permeability. This will lead to releasing of oxygen reactive species with cytochrome $\mathrm{c}$ to the cytoplasm and activation of caspase 9 which, in turn, activates caspase 3 . These actions are considered as the first step in the apoptosis process leading to cell death plus reduction in ATP content of the cell. These are possible causes of proximal tubular cell death and renal injury $[4,5]$. In general, the idea of searching for a drug, medicinal plant or a chemical compound that help protects against VAN-induced nephrotoxicity is well appreciated.

Nigella sativa (NS) is a medicinal plant which is known to have a lot of pharmacological actions, which for most of these actions, antioxidant activity is responsible [6].
NS and its active constituent thymoquinone (TQ) have been found to have a protective effect against drug-induced nephrotoxicities such as thioacetamide [7] cisplatin [8], chemotherapy [9], haloperidol [10], and methotrexate [11].

The aim of the present study, therefore, was to investigate a possible protective effect of NS on VAN-induced nephrotoxicity in rats.

For the best of our knowledge, the present work is the first study designed to unveil the nephroprotective activity of NS oil against VAN-induced nephrotoxicity.

\section{METHODS}

Chemicals

VAN as hydrochloride (1 g vial) was purchased from Julphar Gulf pharmaceutical industries, Ras Al-Khaimah, United Arab Emirate. NS oil was obtained by cold press extraction by a special machine. The opinion of an expert herbalist was taken to ensure authentication of the seed; nonetheless, a voucher specimen of the seed was kept in the Department of Pharmacology as a reference.

\section{Animals}

The protocol of the present study was approved by the Institutional Ethical Committee. Twenty-eight adult male Albino rats weighing 175-350 g were obtained from the animal house of the College of Veterinary Medicine/Basrah University and kept in the animal house of the College of Medicine/Basrah University for 2 weeks for acclimatization in separated cages under controlled conditions with free access to water and standard food. All measures were taken to minimize the suffering of the animals in accordance with the guidelines (National Institutes for Health USA publication, 1985). 
Experimental protocol

The rats were divided into four groups (7 rats) in each as follows:

- Group I - (Control) was treated with normal saline orally $(2 \mathrm{ml} / \mathrm{kg} /$ day $)+$ normal saline $(2 \mathrm{ml} / \mathrm{kg} /$ day) intraperitoneally (IP)

- Group II - was treated with VAN (400 mg/kg/day) IP + normal saline $(2 \mathrm{ml} / \mathrm{kg} /$ day) orally

- Group III - was treated with NS oil orally $(2 \mathrm{ml} / \mathrm{kg} /$ day $)+$ normal saline $(2 \mathrm{ml} / \mathrm{kg} /$ day $) \mathrm{IP}$

- Group IV - (VAN+NS) was treated with NS oil ( $2 \mathrm{ml} / \mathrm{kg} /$ day) orally+ VAN (400 mg/kg/day) IP.

The animals were treated for 7 consecutive days. Twenty-four hours after the last dose, the animals were sacrificed after short anesthesia with diethyl ether. Blood samples were collected directly from the heart and transferred into a tube containing (thrombin). The tubes were centrifuged for $20 \mathrm{~min}$ at $1000 \mathrm{round} / \mathrm{min}$. Serum was obtained and stored at $-20^{\circ} \mathrm{C}$ until assayed. Both kidneys were dissected. One kidney for homogenate preparation and the other was kept in $10 \%$ formalin for histopathological examination.

\section{Laboratory measurements}

\section{Estimation of serum urea and creatinine}

Serum urea and creatinine were measured using a spectrophotometer (INTEGRA machine, Roche (Germany).

\section{Estimation of renal tissue glutathione and malondialdehyde}

- Renal tissue glutathione (GSH) and malondialdehyde (MDA) were measured using MYBIOSOURCE commercial ELISA kit

- (China) Specific for rats. This kit uses competitive -ELISA method.

Estimation of renal tissue neutrophil gelatinase-associated lipocalin Renal tissue neutrophil gelatinase-associated lipocalin (NGAL) concentration was measured using MYBIOSOURCE rat ELISA kit (China). The method is based on sandwich ELISA technology.

\section{Histopathological examination}

The slides were prepared from kidney specimens, stained with Hematoxylin and Eosin (H\&E), coded and blindly examined by an unbiased histopathologist. Histopathological findings were classified according to the severity of changes to score $0,1,2$, and 3 for no change, 0.25 change, 0.50 change, and $>0.50$ change observed in the specimens of the kidneys, respectively.

\section{Statistical analysis}

Statistical Package for Social Sciences version 20 was used. The results were expressed as mean \pm standard deviation. Differences between the groups were estimated using independent samples $t$-test. Statistical significance was considered when value of $\mathrm{p}<0.05$.

\section{RESULTS}

\section{Effect of Nigella sativa on renal function test}

There was a statistically significant increase in the levels of serum urea and creatinine following treatment with VAN as compared to the control, $(p<0.001)$. When VAN and NS were given in combination for 7 days, the levels of serum urea and creatinine significantly decreased compared to VAN treated group, $(\mathrm{p}<0.001)$ (Table 1$)$.
The level of NGAL in renal tissue was increased to $1.026 .84 \pm 682.17 \mathrm{pg} / \mathrm{ml}$ in VAN treated group compared to a level of $770.69 \pm 340.88 \mathrm{pg} / \mathrm{ml}$ in the control group. Treatment with VAN and NS combination lowered NGAL levels toward the control value. None of these changes in NGAL levels obtained statistical significance (Table 1).

\section{Effect of nigella sativa on parameters of oxidative stress}

Effect on renal tissue malondialdehyde

There was a small and insignificant increase in renal tissue MDA in the rats treated with VAN. Treatment with the combination of VAN and NS showed a small and insignificant reduction in renal tissue MDA level. The only significant change in renal tissue MDA levels compared to the control values was observed in NS treatment alone, $(\mathrm{p}<0.033)$ (Table 2).

\section{Effect on renal tissue glutathione}

There was a statistically significant decrease in renal tissue GSH following treatment with VAN compared to the control $(\mathrm{p}<0.0001)$. While treatment with a combination of NS and VAN significantly raised renal tissue GSH toward the control level $(\mathrm{p}<0.0001)$ (Table 2).

\section{Effect of nigella sativa and vancomycin on kidney morphology}

In VAN-treated group, the kidneys looked pale, gray in color, bigger in size and easily detached from the animal body, whereas, in the VAN and NS combination treated group, the kidneys maintained normal, healthy color and firmly attached to the animal body.

Following treatment with VAN at a dose of $400 \mathrm{mg} / \mathrm{kg} /$ day for seven days, the homogenate of kidney tissue and the supernatant obtained following centrifugation of the homogenate looked pale, grey. While treatment with the combination of VAN and NS for seven days has resulted in a light red colour of supernatant which is similar to that of the control. This means that NS corrected the changes in kidney tissues induced by VAN.

\section{Effect of Nigella sativa on kidney weight and length}

The mean weight of the rat kidney was doubled after treatment with VAN. This elevation achieved statistical significance $(p<0.0001)$. Treatment with the VAN and NS combination significantly decreased kidney weight toward the control. This obtained statistical significance $(\mathrm{p}<0.0001)$ (Table 3).

Kidney length, as well, was significantly increased by VAN treatment compared to the control, $\mathrm{p}<0.001$. Treatment with the combination of VAN and NS significantly reduced kidney length toward the control, $\mathrm{p}<0.001$ (Table 3).

\section{Histopathological examination \\ Control group}

No histopathological changes were observed in both the control and NS groups (zero score) (Fig. 1 and Table 4).

\section{Vancomycin group}

All VAN-treated animals showed variable degrees of histopathological changes in kidney specimens (sample size=7). Score 2 was observed in 3 animals, 4 animals were identified to have severe changes (score 3) (Fig. 1 and Table 4).

Table 1: Effect of vancomycin, nigella sativa, and combination of vancomycin and nigella sativa on serum urea, serum creatinine, and renal tissue neutrophil gelatinase-associated lipocaline in rats

\begin{tabular}{llll}
\hline Treatments & Serum urea $(\mathbf{m g} / \mathbf{d l})$ & Serum creatinine $(\mathbf{m g} / \mathbf{d} \mathbf{)}$ & NGAL $(\mathrm{pg} / \mathbf{m l})$ \\
\hline C & $34.02 \pm 6.8$ & $0.53 \pm 0.24$ & $770.69 \pm 340.88$ \\
VAN & $184.61 \pm 77.1^{*}$ & $2.41 \pm 0.1^{*}$ & $1026.84 \pm 682.17$ \\
NS & $37.29 \pm 11.8$ & $0.33 \pm 0.08$ & $774.75 \pm 361.31$ \\
VAN+NS & $59.00 \pm 19.5^{* *}$ & $0.44 \pm 0.11^{* *}$ & $844.64 \pm 308.53$ \\
\hline
\end{tabular}

Mean \pm SD. Sample size $=7, \mathrm{p}<0.05$ is statistically significant. ${ }^{*} \mathrm{p}<0.001$, significantly higher than the corresponding values of the control, ${ }^{* *} \mathrm{p}<0.001$, significantly lower than the corresponding values of VAN treated groups. C: Control, VAN: Vancomycin, NS: Nigella sativa, SD: Standard deviation, NGAL: Neutrophil gelatinase-associated lipocaline, VAN+NS: Vancomycin+nigella sativa 
The observed histopathological changes were as follows:

1. Dilatation of renal tubules

2. Cast formation

3. Flattening of renal tubule lining epithelial cells

4. Hemorrhage

5. Glomerular atrophy

6. Infiltration of inflammatory cells

7. Cyst formation

8. Necrosis

9. Sloughing of the kidney capsule.

\section{Vancomycin + nigella sativa group}

Treatment with NS oil and VAN together resulted in ameliorating the severity of histopathological changes; in 4 animals, there were no

Table 2: Effect of vancomycin, nigella sativa, and combination of vancomycin and nigella sativa on glutathione and malondialdehyde in renal tissue homogenate in rats

\begin{tabular}{lll}
\hline Treatments & GSH $(\mathbf{m g} / \mathbf{m l})$ & MDA $(\mathbf{n g} / \mathbf{m l})$ \\
\hline C & $4.6 \pm 1.2$ & $23 \pm 6.5$ \\
VAN & $2.11 \pm 0.5^{*}$ & $27.9 \pm 4.3$ \\
NS & $3.6 \pm 2.1$ & $15.7 \pm 4.7^{* * *}$ \\
VAN+NS & $4.15 \pm 0.1^{* *}$ & $25.3 \pm 8$ \\
\hline
\end{tabular}

Mean \pm SD. Sample size $=7, \mathrm{p}<0.05$ is statistically significant. ${ }^{*} \mathrm{p}<0.0001$, significantly lower than control, ${ }^{* *} \mathrm{p}<0.0001$, significantly higher than VAN group, ${ }^{* * *} \mathrm{p}<0.001$, significantly lower than control, VAN and VAN+NS values. C: Control, VAN: Vancomycin, NS: Nigella sativa, GSH: Glutathione, MDA: Malondialdehyde, SD: Standard deviation, VAN+NS: Vancomycin+nigella sativa histopathological changes, mild changes were observed in 2 animals and moderate changes were observed in only one animal (Fig. 1 and Table 4).

\section{DISCUSSION}

VAN is regarded as an indispensable antibiotic for the treatment of MRSA, which is one of the most resistant bacteria [1]. This achievement which is accredited for VAN is unfortunately confounded by harmful side effects. One of these side effects is nephrotoxicity which, despite all precautions, it cannot be overcome [2]. NS, a medicinal plant which is frequently reported having a nephroprotective activity against nephrotoxicities induced by several drugs such as methotrexate [11], gentamicin [12], and cisplatin [13]. These facts laid the basis for performing the current study to explore a possible protective effect of NS oil against VAN-induced nephrotoxicity in an animal model. In the current study, VAN was found to have a clear toxicity to the kidney of rats. The kidneys were directly inspected before removal from the animals. The color of the kidneys in VAN-treated animals, appeared pale gray-to-pink in color with a double increase in kidney weight, in addition, the kidneys were easily detached from the animals. A possible explanation for these changes in kidney color could be attributed to renal tubular ischemia caused by VAN [14], and the increased kidney volume and weight could be due to edema. A similar observation of increased kidney weight and volume was also reported by Takigawa et al. [15]. On the contrary, Bayomy found that administration of VAN in rats caused a significant reduction in rats' body and kidney weight. The author attributed these changes to anorexia, low food intake and high levels of catabolism as a result of acute renal failure and acidosis [16].

These findings suggest that the kidneys were severely damaged by VAN treatment. A similar result was also observed by

Table 3: Effect of Vancomycin, nigella sativa and combination of vancomycin and nigella sativa on animal weight, kidney weight, and length

\begin{tabular}{lllll}
\hline Parameters & Animal weight before treatment $\mathbf{( g )}$ & Animal weight after treatment (g) & Kidney weight (g) & Kidney length (mm) \\
\hline C & $247.6 \pm 63$ & $258.9 \pm 63$ & $0.92 \pm 0.14$ & $16.9 \pm 1$ \\
VAN & $273.3 \pm 46$ & $284.9 \pm 47$ & $2.19 \pm 0.48^{*}$ & $20.9 \pm 2.3^{*}$ \\
NS & $230.9 \pm 50$ & $243.3 \pm 49$ & $0.75 \pm 0.13$ & $16.1 \pm 1.5$ \\
VAN+NS & $221.9 \pm 45$ & $237.4 \pm 48$ & $0.87 \pm 0.29^{* *}$ & $16.9 \pm 1.6^{* *}$ \\
\hline
\end{tabular}

Mean \pm SD. Sample size $=7, \mathrm{p}<0.05$ is statistically significant. ${ }^{*} \mathrm{p}<0.0001$ significantly higher than the corresponding values of the control. ${ }^{* *} \mathrm{p}<0.02$ significantly lower than the corresponding values of a VAN. C: Control, VAN: Vancomycin, NS: Nigella sativa, SD: Standard deviation, VAN+NS: Vancomycin+nigella sativa

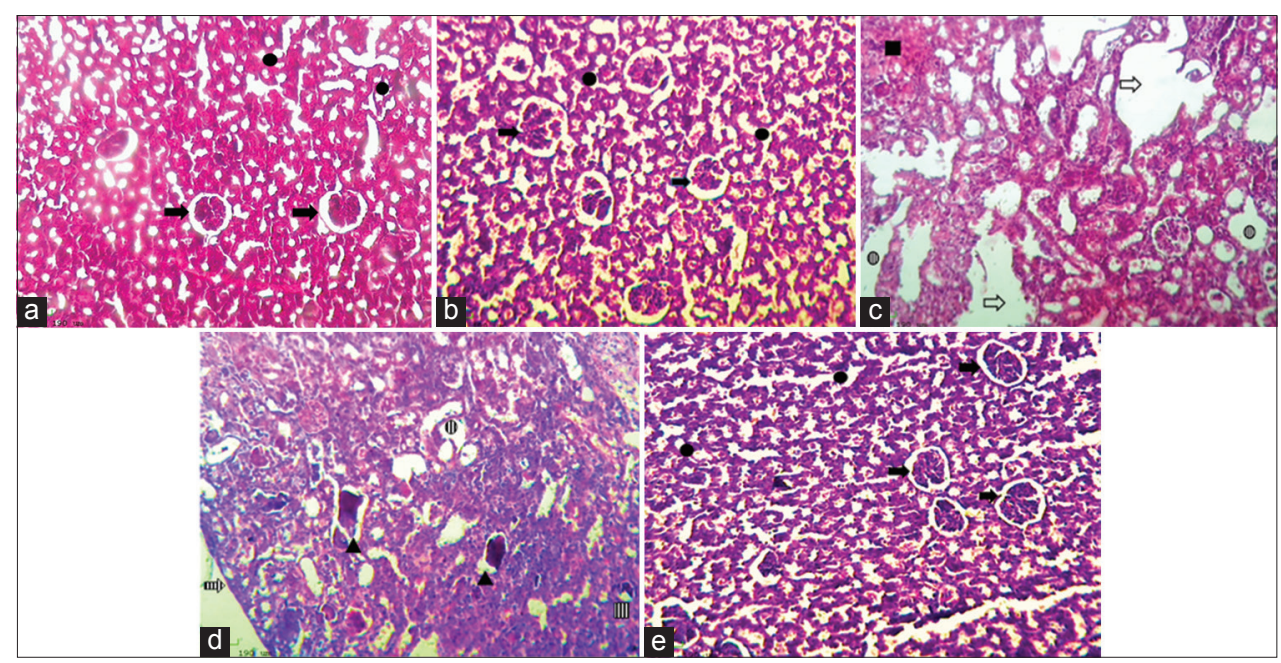

Figure 1: Representative slides of rat kidney revealing the effect of the treatment with the following compounds for 7 days: (a) normal saline $2 \mathrm{ml} / \mathrm{kg} /$ day (Control), (b) nigella sativa oil $2 \mathrm{ml} / \mathrm{kg} / \mathrm{day}$, (c and d) vancomycin $400 \mathrm{mg} / \mathrm{kg} / \mathrm{day}$ and (e) nigella sativa oil +

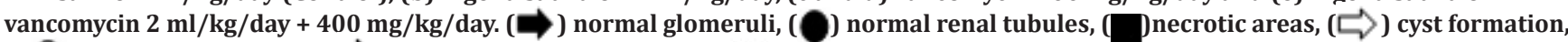
(1I) )dilated renal tubules, (ㄷ) )sloughing of kidney capsule, ( $\boldsymbol{\Delta}$ ) cast formation, (而) glomerular atrophy. All slides are stained with $\mathrm{H}$ and $\mathrm{E}$, and presented as a power of $\times 10$ 
Table 4: Histopathological score of renal damage induced by nigella sativa, vancomycin and combination of vancomycin and nigella sativa

\begin{tabular}{lllll}
\hline \multirow{2}{*}{ Scores } & \multicolumn{3}{l}{ Number of animals } \\
\cline { 2 - 5 } & Control & NS & VAN & NS+VAN \\
\hline 0 & 7 & 7 & 0 & 4 \\
1 & 0 & 0 & 0 & 2 \\
2 & 0 & 0 & 3 & 1 \\
3 & 0 & 0 & 4 & 0 \\
\hline
\end{tabular}

Score $0,1,2$ and 3 are for normal, mild, moderate, and sever histopathological changes in renal tissue, respectively. Sample size $=7$. NS: Nigella sativa, VAN:Vancomycin, VAN+NS: Vancomycin+nigella sativa

Naghibi et al. [17]. These changes were supported by histopathological examination. Histopathological changes were labeled as mild, moderate, and severe which characterized by dilatation in renal tubules with desquamation of renal tubular epithelial cells and cyst formation with the cast and the proliferation of inflammatory cells of renal tissues. The fact that nephrotoxicity was seen in all VAN treated animals made an evaluation of the effect produced by NS feasible; which means any effect which can be observed with NS is taken as a true effect. NS markedly ameliorated toxicity produced by the VAN. Histopathological changes have decreased significantly and also the levels of serum urea and creatinine declined significantly. NGAL was chosen for the present study as a valuable biomarker, more specific than serum creatinine and can discover early changes in acute tubular injury [18]. In the current study, changes in renal tissue NGAL following treatment with VAN were small and the effect of NS was small too. Therefore, a conclusive result cannot be satisfied.

These results are highly suggestive of the protective effect of NS in VAN-induced nephrotoxicity. In addition, the group of animals treated with VAN and NS, the kidneys returned to its normal color and the weight of the kidneys returned to that of the control group.

The observed nephrotoxic effect of VAN could involve oxidative stress since VAN adversely affects the parameters of oxidative stress (MDA and GSH) in the homogenate of kidney tissue. Despite decades of interest in VAN-induced nephrotoxicity, the underlying mechanism is still not well defined. In one study, the expression of the gene was studied to explore the most probable mechanism of VAN-induced nephrotoxicity [19]. This study has substantiated the importance of gene markers of toxicity of the kidney, but at the same time, this study could not eliminate the involvement of inflammation, necrosis and oxidative stress of proximal tubular cells as mechanisms for VAN-induced renal injury [19].

NS was found to have a renoprotective effect because of its anti-inflammatory and antioxidant properties. This effect is attributed to the active ingredients $\mathrm{TQ}$, carvacol, and others [6]. In the present study, parameters of oxidative stress, MDA and GSH were evaluated in the homogenate of kidney tissue. Blood samples were not sufficient to perform the analysis on these parameters in the serum. However, there is an advantage to evaluate these parameters in target tissues since this reflects direct changes. There is evidence suggesting that oxidative stress plays a major role in VAN-induced nephrotoxicity and tubular damage and the reversal of oxidative stress with a thiol derivative mucolytic agent (erdosteine) reduces VAN-induced nephrotoxicity [20].

In the current study, administration of VAN at a dose of $400 \mathrm{mg} / \mathrm{kg} /$ day led to an increase in MDA level in the homogenate of kidney tissue which indicates the role of oxidative stress in renal damage due to lipid peroxidation; this result is in agreement with other studies [16,20]. On the other hand, administration of NS oil has resulted in a reduction in MDA level in kidney tissues which indicates its oxidative vanquishing ability and this is consistent with the study of $\mathrm{Uz}$ et al. [21].

GSH is an important antioxidant system in the cell which is responsible for the protection of the cell against intracellular free radicals, peroxides and toxic compounds by conjugation with toxic substances [22]. Depletion of GSH in renal tissues is considered as one of the major factors that permit lipid peroxidation [23]. In the current study, treatment with VAN resulted in a significant decrease in GSH renal tissue's level. This indicates renal injury due to oxygen free radical formation which depletes GSH defense system. This result is compatible with the results of Ahmida [23]. On the other hand, concomitant administration of NS oil with VAN has resulted in a significant elevation of GSH concentration in renal tissue. This effect confirms the antioxidant potential of NS and in addition may explain the protective effect of NS in VAN-induced nephrotoxicity. In one study, it has been observed that TQ, which is the active constituent of NS, caused an elevation in renal tissue GSH level in rats treated concomitantly with sodium nitrite and protect the animal renal system from injury. TQ is the most powerful antioxidant, antiinflammatory, and anti-apoptotic ingredient present in NS [24].

\section{CONCLUSION}

The present study demonstrates that NS has a nephroprotective effect against VAN-induced nephrotoxicity. This effect is attributed to the antioxidant potential inherited in NS.

\section{ACKNOWLEDGMENT}

The authors would like to thanks Dr. Zainab W. Khudair, Professor at the Pathology Department in the College of Veterinary Medicine, University of Basrah, for performing histopathological examination of this study and to the staff of the Department of Pharmacology, College of Pharmacy, University of Basrah, for technical assistance and support.

\section{AUTHORS' CONTRIBUTIONS}

1. Conception or design: Jawad H Ahmed

2. Data collection and processing: Sarah T Omran

3. Analysis and interpretation of the data: Jawad H Ahmed, Sarah T Omran

4. Writing substantial sections of the paper (e.g.synthesizing findings in the literature review or the findings/results section): Jawad $\mathrm{H}$ Ahmed, Sarah T Omran.

\section{CONFLICT OF INTEREST}

None declared by the authors.

\section{REFERENCES}

1. Mansour H, Bush JA, Brito M, Novotny P, Courtney C, Mustonen J, et al. The effect of vancomycin doses greater than 2 grams on serum creatinine and vancomycin trough levels. Int $\mathrm{J}$ Pharm Pharm Sci 2014;6:621-5.

2. Mergenhagen KA, Borton AR. Vancomycin nephrotoxicity: A review. J Pharm Pract 2014;27:545-53.

3. Stokes MB. Vancomycin in the kidney-a novel cast nephropathy. J Am Soc Nephrol 2017;28:1669-70.

4. Bamgbola O. Review of vancomycin-induced renal toxicity: An update. Ther Adv Endocrinol Metab 2016;7:136-47.

5. Sakamoto Y, Yano T, Hanada Y, Takeshita A, Inagaki F, Masuda S, et al. Vancomycin induces reactive oxygen species-dependent apoptosis via mitochondrial cardiolipin peroxidation in renal tubular epithelial cells. Eur J Pharmacol 2017;800:48-56.

6. Alotaibi FO, Mustafa G, Ahuja AL. A study of the enhanced antiinflammatory potential of nigella sativa in topical nanoformulation. Int J Pharm Pharm Sci 2018;10:41-51.

7. Ahmad A, Al-Abbasi FA, Sadath S, Ali SS, Abuzinadah MF, Alhadrami HA, et al. Ameliorative effect of camel's milk and Nigella sativa oil against thioacetamide-induced hepatorenal damage in rats. Pharmacogn Mag 2018;14:27-35

8. Hosseinian S, Hadjzadeh MA, Roshan NM, Khazaei M, Shahraki S, Mohebbati R, et al. Renoprotective effect of Nigella sativa against cisplatin-induced nephrotoxicity and oxidative stress in rat. Saudi J Kidney Dis Transpl 2018;29:19-29.

9. Cascella M, Palma G, Barbieri A, Bimonte S, Amruthraj NJ, Muzio MR, et al. Role of Nigella sativa and its constituent thymoquinone on chemotherapy-induced nephrotoxicity: Evidences from experimental animal studies. Nutrients 2017;9:E625.

10. Akintunde JK, Abubakar OK. Novel therapeutic approaches of natural 
oil from black seeds and its underlying mechanisms against kidney dysfunctions in haloperidol-induced male rats. Drug Metab Pers Ther 2017;32:97-107.

11. Ahmed JH, Abdulmajeed IM. Effect of Nigella sativa (black seeds) against methotrexate-induced nephrotoxicity in mice. J Intercult Ethnopharmacol 2017;6:9-13.

12. Saleem U, Ahmad B, Rehman K, Mahmood S, Alam M, Erum A, et al. Nephro-protective effect of Vitamin C and Nigella sativa oil on gentamicin associated nephrotoxicity in rabbits. Pak J Pharm Sci 2012;25:727-30.

13. Farooqui Z, Shahid F, Khan AA, Khan F. Oral administration of Nigella sativa oil and thymoquinone attenuates long term cisplatin treatment induced toxicity and oxidative damage in rat kidney. Biomed Pharmacother 2017;96:912-23.

14. Humanes B, Jado JC, Camano S, Lopez-Parra V, Torres AM, Al varezSala LA, et al. Protective effects of cilastatin against vancomycininduced nephrotoxicity. Biomed Res Int 2015;2015:704382.

15. Takigawa M, Masutomi H, Kishimoto Y, Shimazaki Y, Hamano Y, Kondo Y, et al. Time-dependent alterations of vancomycin-induced nephrotoxicity in mice. Biol Pharm Bull 2017;40:975-83.

16. Bayomy NA, Abdelaziz EZ, Said MA, Badawi MS, El-Bakary RH. Effect of pycnogenol and spirulina on vancomycin-induced renal cortical oxidative stress, apoptosis, and autophagy in adult male albino rat. Can J Physiol Pharmacol 2016;94:838-48.

17. Naghibi B, Ghafghazi T, Hajhashemi V, Talebi A. Vancomycin-induced nephrotoxicity in rats: Is enzyme elevation a consistent finding in tubular injury? J Nephrol 2007;20:482-8.
18. Moledina DG, Hall IE, Thiessen-Philbrook H, Reese PP, Weng FL, Schröppel B, et al. Performance of serum creatinine and kidney injury biomarkers for diagnosing histologic acute tubular injury. Am J Kidney Dis 2017;70:807-16.

19. Dieterich C, Puey A, Lin S, Swezey R, Furimsky A, Fairchild D, et al. Gene expression analysis reveals new possible mechanisms of vancomycin-induced nephrotoxicity and identifies gene markers candidates. Toxicol Sci 2009; 107:258-69.

20. Oktem F, Arslan MK, Ozguner F, Candir O, Yilmaz HR, Ciris M, et al. In vivo evidences suggesting the role of oxidative stress in pathogenesis of vancomycin-induced nephrotoxicity: Protection by erdosteine. Toxicology 2005;215:227-33.

21. Uz E, Bayrak O, Uz E, Kaya A, Bayrak R, Uz B, et al. Nigella sativa oil for prevention of chronic cyclosporine nephrotoxicity: An experimental model. Am J Nephrol 2008;28:517-22.

22. Chakraborty SP, Mahapatra SK, Roy S. In vitro time-dependent vancomycin-resistant Staphylococcus aureus-induced free radical generation and status of antioxidant enzymes in murine peritoneal macrophage. Toxicol Mech Methods 2012;22:9-22.

23. Ahmida MH. Protective role of curcumin in nephrotoxic oxidative damage induced by vancomycin in rats. Exp Toxicol Pathol 2012;64:149-53.

24. Elsherbiny NM, Maysarah NM, El-Sherbiny M, Al-Gayyar MM. Renal protective effects of thymoquinone against sodium nitrite-induced chronic toxicity in rats: Impact on inflammation and apoptosis. Life Sci 2017;180:1-8 